\title{
Vulnerabilidade e envelhecimento no contexto da saúde
}

\author{
Vulnerability and aging in the bealth context
}

Vulnerabilidad y envejecimiento en el contexto de la salud

\section{RESUMO}

\section{Adriana Aparecida Paz ${ }^{1}$ Beatriz Regina Lara dos Santos², Olga Rosaria Eidt ${ }^{3}$}

O envelhecimento da população associa-se a importantes transformações sociais e econômicas, bem como à mudança no perfil epidemiológico e, conseqüentemente, nas demandas dos serviços de saúde. Nessa perspectiva, realizou-se este estudo, buscando, a partir da pesquisa bibliográfica das áreas temáticas envelhecimento e vulnerabilidade, identificar os elementos que contribuem para a vulnerabilidade individual, social e programática das pessoas idosas. Realizou-se uma incursão pela literatura, sendo selecionadas as bibliografias temáticas para envelhecimento e vulnerabilidade. Com a visão abrangente, apropriada desses conceitos, é possível propor uma reconstrução ampliada e reflexiva das práticas em saúde relacionadas à prevenção de agravos e promoção da saúde, visto que os problemas-alvo são as suscetibilidades populacionais que demandam respostas sociais e profissionais.

Descritores: Vulnerabilidade em saúde; Idoso; Saúde do idoso

\begin{abstract}
The aging of the population is associated with important social and economic changes, as well as with changes in the epidemiological profile and demands of public health services. Therefore, the purpose of this study was to identify factors that contributed to individual, social, and programmatic vulnerability of the elderly people. A qualitative, descriptive study was carried out through a systematic review and synthesis of the literature about aging and vulnerability. The vulnerability of the aging population requires appropriate social and professional responses. There is a need to reflect upon health practices related to the prevention of injuries and promotion of health of the aging population.
\end{abstract}

Descriptores: Health; Vulnerability; Elderly; Elder Health.

\section{RESUMEN}

El envejecimiento de la población se asocia a importantes transformaciones sociales y económicas así como también a importantes cambios en el perfil epidemiológico y consecuentemente, en las demandas de los servicios de salud. En esa perspectiva, se realizó un estudio, buscando identificar a partir de la investigación bibliográfica de las áreas temáticas envejecimiento y vulnerabilidad, los elementos que contribuyen a la vulnerabilidad individual, social y programática de las personas ancianas. Se incursionó en la literatura, siendo seleccionadas las bibliografías temáticas para envejecimiento y vulnerabilidad. Con la visión amplia, apropiada de esos conceptos, es posible proponer una reconstrucción reflexiva de las prácticas en salud relacionadas a la prevención de afecciones y promoción de la salud, dado que los problemas blanco son las susceptibilidades poblacionales que demandan respuestas sociales y profesionales.

Descritores: Vulnerabilidad en salud; Anciano; Salud del anciano

\footnotetext{
${ }^{1}$ Mestre em Enfermagem; Professor ano Curso de Bacharelado em Enfermagem da Faculdade Fátima (FATIMA); e da Faculdade Cenecista de Bento Gonçalves - FACEBG - Bento Gonçalves (RS), Brasil.

${ }^{2}$ Doutora em Educação; Professora na Faculdade de Enfermagem, Fisioterapia e Nutrição - FAENFI/UFRGS; Coordenadora do Núcleo Interdisciplinar de Pesquisas e Estudos em Promoção e Vigilância da Saúde - NIPEPROVIS/PUCRS - .

${ }^{3}$ Doutora em Enfermagem Pediátrica; Professora Colaboradora na Escola de Enfermagem da Universidade Federal do Rio Grande do Sul EEUFRGS Porto Alegre (RS), Brasil; Professora na Faculdade de Enfermagem, Fisioterapia e Nutrição, Pontifícia Universidade Católica do Rio Grande do Sul FAENFI/UFRGS; Vice-Coordenadora do Núcleo Interdisciplinar de Pesquisas e Estudos em Promoção e Vigilância da Saúde NIPEPROVIS/PUCRS - Porto Alegre (RS), Brasil.
} 


\section{INTRODUÇÃO}

A vulnerabilidade é um constructo conceitual, o qual se revela um instrumento de inteligibilidade de situações de saúde e seus determinantes. Envolve os planos biológico/individual, social e programático/ institucional ${ }^{(1)}$, considerados como um todo indivisível. Conseqüentemente, qualquer mudança em um dos planos sempre ocasionará conseqüências nos demais. Assim, a vulnerabilidade busca a "universalidade não na reprodutibilidade ampliada de sua fenomenologia e inferências, mas no interesse e possibilidade 'transregionais' de sua pragmática"(2).

O caráter não-probabilístico da vulnerabilidade a torna interessante pelo fato de ser aplicável a qualquer dano ou condição de interesse para a saúde pública, superando, portanto, a tradicional abordagem das estratégias de redução de risco empregada pela epidemiologia, pois a grande pretensão é a busca da síntese dos três planos individual, social e programático ${ }^{(2)}$, planos esses descritos pelos autores e adotados no estudo.

O aumento da população idosa no Brasil apresenta um crescimento progressivo e rápido. No ano de 2000 , entre 169 milhões de habitantes, 8,6\% eram idosos. O Estado do Rio Grande do Sul ocupa o segundo lugar no cenário brasileiro, tendo, entre sua população total, 10,5\% de idosos. Esses dados mostram-se muito expressivos e denotam que a sociedade necessita de subsídios e aprimoramentos para atuar em benefício dessa população crescente $^{(3)}$.

O envelhecimento da população associa-se a importantes transformações sociais e econômicas, bem como à mudança no perfil epidemiológico e demandas dos serviços de saúde. Tal mudança, no Brasil, implica elevação dos custos diretos e indiretos para o sistema de saúde ${ }^{(3-5)}$, fazendo do envelhecimento um fenomeno que precisa de ampla discussão. Portanto, o aumento da longevidade e os aspectos a ela inerentes fazem o fenômeno do envelhecimento constituir uma questão atual. Sendo assim, este estudo tem como finalidade fornecer subsídios para a gestão dos serviços de saúde e para as ações de prevenção e controle de agravos à saúde, a partir do objetivo identificar os elementos que contribuem para a vulnerabilidade da população idosa brasileira, por meio de uma pesquisa bibliográfica das áreas temáticas envelhecimento e vulnerabilidade.

\section{CONTEXTUALIZANDO A VULNERABILI- DADE DA PESSOA IDOSA}

No Brasil, a atenção à saúde do idoso é uma especialidade em expansão que ainda carece de profissionais. Tal atenção deve oportunizar o cuidado integral e contextualizado, através do reconhecimento de necessidades e de aspectos individuais e coletivos dessa população( ${ }^{()}$. Assim, aborda-se aspectos relacionados às dimensões da vulnerabilidade, visando subsidiar políticas de prevenção de doenças e práticas de cuidado individual e coletivo.

\section{Vulnerabilidade biológica/individual no envelhecimento}

A vulnerabilidade individual ${ }^{(2)}$ refere-se "ao grau e à qualidade da informação de que os indivíduos dispõem sobre o problema; à capacidade de elaborar essas informações e incorporá-las aos seus repertórios cotidianos [...], ao interesse e às possibilidades efetivas de transformar essas preocupações em práticas protegidas e protetoras". Portanto, vulnerabilidade individual é o que uma pessoa, na sua singularidade, pensa, faz e quer, e o que, ao mesmo tempo, a expõe ou não à aquisição de um agravo à saúde. Refere-se à idade, à hereditariedade, assim como ao tipo de informação de que a pessoa dispõe, e de como a utiliza.

As alterações biológicas tornam o idoso menos capaz de manter a homeostase quando submetido a um estresse fisiológico. Tais modificações, principalmente quando associadas à idade cronológica avançada, determinam maior suscetibilidade à ação de doenças, crescente vulnerabilidade e maior probabilidade de morte ${ }^{(3)}$. Atualmente, no Brasil, observa-se a queda acentuada da mortalidade infantil, redução da mortalidade por doenças infecto-contagiosas e um aumento significativo da mortalidade por doenças crônicas não-transmissíveis, ${ }^{(3,7)}$

Embora esta doenças não sejam exclusivas das faixas etárias mais avançadas, em 1999, entre as causas de óbitos mais freqüentes entre os idosos, identificou-se que 36,9\% correspondiam a doenças do aparelho circulatório, seguidas pelas neoplasias (14,0\%); doenças do aparelho respiratório $(12,2 \%)$; e doenças endócrinas, metabólicas e nutricionais $(5,8 \%)^{(3)}$. Tais doenças, quando associadas à hospitalização, favorecem o declínio funcional e, conseqüentemente, encaminham-se para o desenvolvimento de incapacidades. A hospitalização e o repouso no leito prolongado, de forma intermitente, podem determinar o agravamento da situação de saúde do idoso, tornando-o mais frágil. O declínio da capacidade funcional geralmente conduz a pessoa idosa à limitação ou perda total da capacidade de desempenhar, de forma independente, suas atividades cotidianas.

Geralmente, as atividades instrumentais da vida diária (AIVD's) são atingidas primeiramente, sendo essas utilizadas como indicadores para detectar a incapacidade funcional ${ }^{(8)}$. Essas atividades estão relacionadas a participação social do idoso ou à manutenção de sua integração com o meio social que requer uma reorganização familiar. A partir de incapacidades instaladas 
nas AIVD's, as outras atividades básicas da vida diária (AVD's) também poderão estar comprometidas. A condição de dependência severa para tais atividades foi constatada em 50,0\% dos idosos em condição de alta hospitalar, em 2002, num hospital universitário, do município de Porto Alegre ${ }^{(9)}$. Em 2003, em outro hospital universitário da mesma localidade, um estudo com 164 pessoas idosas em condição de alta hospitalar, constatam que $78,0 \%$ apresentavam dependência para as AIVD's ${ }^{(8)}$.

\section{Vulnerabilidade social do idoso}

Em alguns contextos históricos houve a atribuição de poderes à pessoa conforme seu ciclo vital. Logo, a figura da pessoa idosa, nesses mesmos contextos históricos sofre um intenso "desinvestimento" social e político ${ }^{(10)}$. No entanto, reconhece-se que o aumento da longevidade é uma conquista social que marcou o século XX em quase todo o mundo, mas tal conquista gerou impactos e novas demandas para o Sistema de Saúde e para a família ${ }^{(11-12)}$.

A vulnerabilidade social ${ }^{(2)}$ é atribuída ao modo de "obtenção de informações [... ], como acesso aos meios de comunicação, escolaridade, disponibilidade de recursos materiais, poder de influenciar decisões políticas, possibilidades de enfrentar barreiras culturais, estar livre de coerções violentas ou poder defender-se delas", bem como todos os aspectos referentes à estrutura, à organização e à dinâmica familiar. Assim, as condições culturais, econômicas e políticas precisam ser consideradas, quando se deseja compreender as razões pelas quais as pessoas pensam, fazem e querem coisas que as expõem a um agravo de longa duração ou a eventos não condizentes à qualidade de vida.

A vulnerabilidade social do idoso decorre da diversidade de circunstâncias enfrentadas no cotidiano pela população envelhecida. Tais circunstâncias referem-se aos aspectos relacionados a questões culturais, sociais, econômicas, de saúde, entre outros ${ }^{(13)}$. Por sua vez, quando a pessoa idosa está em situação conjugal de viuvez, essa condição é diferente entre os sexos. A mulher tem a tendência de permanecer só, enquanto o homem geralmente se casa novamente. No Brasil, o baixo valor das aposentadorias constitui uma vulnerabilidade social, pois expõe não apenas a pessoa idosa, mas todo o grupo familiar que sobrevive com esse recurso. As baixíssimas aposentadorias não conseguem promover condição social adequada, ao alcance da qualidade de vida.

Quanto ao idoso portador de morbidade acompanhada de declínio funcional e grau de dependência elevada, constata-se a necessidade de um cuidador para auxiliá-lo nas AIVD's, bem como no atendimento a suas necessidades básicas, o que remete a família a uma nova divisão de papéis e funções para o exercício do cuidado $^{(11,14)}$. Geralmente, esse cuidado é realizado por algum familiar, muitas vezes também idoso, que assume várias tarefas do cotidiano domiciliar.

$\mathrm{Na}$ sociedade atual, algumas atividades colocam os jovens na situação de excluídos ou têm oportunidades limitadas no que se refere, principalmente, ao emprego. As elevadas taxas de desemprego e subempregos, muitas vezes os condicionam a participarem da economia informal $^{(15)}$. Paralelamente, os jovens estão expostos à violência, à criminalidade e à gravidez precoce. Assim, questiona-se se o jovem, ao construir sua vida permeada pela exclusão social, conseguirá ter inclusão (justiça) em sua velhice?

\section{Vulnerabilidade à atenção programática da pessoa idosa}

O Sistema Único de Saúde preconiza a universalização do acesso, a integralidade da atenção, a eqüidade, a descentralização da gestão, a hierarquização dos serviços e o controle social. Assim, a implantação desse sistema pressupõe a reorganização das práticas sanitárias e, conseqüentemente, a transformação do modelo assistencial e da organização do serviço no atendimento à saúde do idoso.

Entre os elementos de organização dos serviços destaca-se a vulnerabilidade programática. O plano programático $^{(2)}$ engloba o acesso e a forma de organização dos serviços de saúde. Inclui, ainda, programas voltados à prevenção, à assistência e ao controle. Seu planejamento e execução ocorrem em níveis nacional, regional ou local, e os seus executores são os governos municipais, estaduais e federal, ou até mesmo as organizações não-governamentais. As instituições consideradas referem-se à saúde, educação, comunidade, cidade, entre outras. Portanto, a vulnerabilidade programática $^{(2)}$ reporta-se aos recursos sociais de que as pessoas necessitam para não se exporem aos agravos e "se protegerem de seus danos que sejam disponibilizados de modo efetivo e democrático". Tal vulnerabilidade refere-se ao grau de alerta e preocupação com o problema, quanto ao modo que se organiza para enfrentá-lo (planejamento, recursos, capacidade, gerência e avaliação), que impede ou limita a intervenção sobre as razões sociais que levam os indivíduos aos modos de pensar, fazer e querer que os expõem aos agravos.

É indiscutível a presença da violência contra o idoso nos âmbitos domiciliar e institucional. Entretanto, no contexto social, constatam-se espaços de violência no que se refere à omissão do Estado na oferta e na avaliação de instituições que oferecem assistência aos idosos ${ }^{(10)}$. Assim, torna-se extremamente necessário incluir todas as formas de intervenção na sociedade, a partir da vigilância à saúde, da proteção e da educação em saúde, tendo como parceiros a família, os profissionais e o 
Estado. Como é mencionado:

os indices desenvolvidos para avaliar vulnerabilidade programática buscam dar conta justamente deste gradiente de permeabilidade e sensibilidade das estruturas sociopoliticas mais abrangentes e as necessidades concretamente postas para a saúde de individuos/população $[\ldots]^{(1)}$.

Diante de tal constructo conceitual, um dos objetivos do Estatuto do Idoso $^{(16)}$ é manter a pessoa idosa na comunidade, junto de sua família, de forma digna e confortável. Muitas vezes, as condições de saúde dos idosos exigem hospitalização; porém, espera-se que essa não tenha longa duração, bem como que a família seja orientada para o cuidado no contexto domiciliar. Entretanto, nas instituições hospitalares do Brasil ${ }^{(17)}$, observa-se a vulnerabilidade programática no que se refere à falta de preparo dos profissionais de saúde para orientar o idoso e o seu cuidador no momento da alta hospitalar.

O envolvimento do idoso, do cuidador/família, da comunidade e dos profissionais de saúde torna-se imprescindível para a elaboração, a efetivação e a avaliação do plano de cuidados após a alta hospitalar ${ }^{(18)}$. Outro ponto a ser destacado é a importância do cuidado de enfermagem domiciliar e as visitas domiciliares realizadas por enfermeiros do Programa de Saúde da Família , bem como o fornecimento de subsídios para estruturação de programas de cuidado domiciliar que atendam à demanda de pacientes idosos.

Portanto, a dependência do idoso ${ }^{(19)}$ é um processo dinâmico e "deve ser abordada por intermédio de programas que incluam estratégias de promoção da saúde até o estabelecimento de redes de apoio aos cuidados de longa duração na comunidade". Da mesma forma, a inexistência de uma política ${ }^{(11)}$ no que se refere aos papéis atribuídos às famílias e aos apoios sociais necessita que rede de serviços ofereçam atenção integral ao idoso com comprometimento funcional.

\section{ESTRATÉGIAS RELACIONADAS Á VULNERABILIDADE E AO ENVELHE- CIMENTO: ALGUMAS CONSIDERAÇÕES}

O envelhecimento, principalmente com dependência ${ }^{(19)}$, é um desafio a ser incorporado pela Saúde Pública, em que se contrapõem duas situações. A primeira refere-se às condições necessárias para a manutenção dos cuidados ao idoso na comunidade, e a segunda refere-se às condições da família para que possa responsabilizar-se pela assistência ao idoso. Portanto, a vulnerabilidade da pessoa idosa não pode ser vista de forma única em cada plano, mas como uma vulnerabilidade constituída desses três planos apresentados. Ainda não se conta com instrumentos que meçam o grau de vulnerabilidade do idoso, sendo este estudo uma oportunidade de teorizar sobre este novo enfoque a ser desvelado. Portanto, a abordagem da vulnerabilidade ${ }^{(2)}$ pressupõe que a mudança não ocorra a partir da pessoa em relação ao outro e seu entorno, mas que seja construída nas possibilidades das pessoas estarem diante de outras, de modo a tornar a saúde de todos mais satisfatória.

Conhecer a vulnerabilidade de grupos populacionais possibilita mobilizar profissionais e população civil, por meio de um processo educativo construtivista, para transformações sociais. Tais transformações devem ser alicerçadas nas relações intersetoriais e na ação comunicativa entre os sujeitos sociais. Assim, acreditase na importância de diferentes formas de enfrentamento, em termos não somente assistencial, de tratamento clínico e de reabilitação, mas também na implementação de políticas públicas e de ações de prevenção de doenças, bem como promoção de saúde da população de forma integral e resolutiva.

Desse modo, quanto maior o grau de compromisso, a qualidade, os recursos, a gerência e o monitoramento de programas nacionais, regionais e locais de prevenção e de cuidado ao idoso, maiores serão as chances de canalizar os recursos sociais existentes, otimizar seu uso e identificar a necessidade de outros recursos, fortalecendo "as pessoas idosas diante dos agravos de longa duração e suas conseqüências"(2). Frente a tal concepção conceitual de vulnerabilidade, será possível propor uma reconstrução ampliada e reflexiva das práticas em saúde relacionadas à promoção e proteção da saúde da população, visto que os problemas-alvo são as suscetibilidades populacionais, que resultam numa resposta social.

\section{REFERÊNCIAS}

1. Ayres JRCM, França Junior I, Calazans GJ. AIDS, vulnerabilidade e prevenção. In : II Seminário Saúde Reprodutiva em Tempos de AIDS; 1997 Out 2-7; Rio de Janeiro: IMS/UERJ - ABIA; 1997. p. 20-37.

2. Ayres JRCM, França Junior I, Calazans GJ, Saletti Filho HC. O conceito de vulnerabilidade e as práticas de saúde: novas perspectivas e desafios. In: Czeresnia D, Freitas CM. Promoção da saúde: conceitos, reflexões, tendências. Rio de Janeiro: Fiocruz; 2003. p. 117-39.

3. Anuário estatístico de saúde do Brasil 2001 [texto na Internet]. Brasília (DF); Ministério da Saúde; 2002. [citado 2003 Fev 9]. Disponível em: http:/ / portal.saude.gov.br/ saude/aplicações/anuario2001/index.cfm

4. Chaimowicz F. A saúde dos idosos brasileiros às vésperas do século XXI: problemas, projeções e alternativas. Rev Saúde Pública = J Public Health.1997; 31(2):184-200.

5. Vermelho LL, Monteiro MFG. Transição demográfica e epidemiológica. In: Medronho RA, Carvalho DM, Bloch 
KV, Luiz RR, Werneck GL, editores. Epidemiologia. São Paulo: Atheneu; 2002. p. 91-103.

6. Llamazares MAM. La vejez y la enfermedad crónica. In: Serrano González MI. Educación para la salud del siglo XXI. Comunicación y salud. Madrid: Editorial Díaz de Santos; 1999. p. 223-9.

7. Santos SSC. Enfermagem gerontogeriátrica: reflexão à ação cuidativa. 2a ed. São Paulo: Robe Editorial; 2001.

8. Paz AA. Características de pessoas idosas em condição de alta hospitalar associadas à rehospitalização [tese] . Porto Alegre (RS): Escola de Enfermagem da UFRGS; 2004.

9. Pazinatto MC. Características clínicas e funcionais do paciente idoso que recebe alta hospitalar e suas necessidades de cuidados após a alta [tese]. Porto Alegre (RS): Instituto de Geriatria e Gerontologia da PUCRS; 2003.

10. Minayo MCS. Violência contra idosos: relevância para um velho problema. Cad Saúde Pública $=$ Rep Public Health. 2003; 19(3):783-91.

11. Karsch UM. Idosos dependentes: famílias e cuidadores. Cad Saúde Pública = Rep Public Health. 2003; 19(3):861-6.

12. Lehr U. A revolução da longevidade: impacto na sociedade, na família e no indivíduo. Estud Interdiscipl Envelhec. 1999;
1:7-35.

13. Cantera IR, Domingo PL. Guias práticos de enfermagem. Rio de Janeiro: McGraw Hill Interamericana do Brasil; 1998.

14. Duarte MJRS. Atenção ao idoso: um problema de saúde e de enfermagem. Rev Enfermagem UERJ. 1994; 2(1):100-11.

15. Camarano AA. Envelhecimento da população brasileira: uma contribuição demográfica. In: Freitas EV, Py L, Neri AL, Cançado FAX, Gorzoni ML, Rocha SM. Tratado de geriatria e gerontologia. Rio de Janeiro: Guanabara Koogan; 2002. p. 58-71.

16. Estatuto do idoso [texto na Internet]. Brasília (DF): Senado Federal; 2003. [citado 2003 Out 17]. Disponível em: http:/ /www.direitodoidoso.com.br/05/estatuto_do_idoso.pdf

17. Karsch UMS, Leal MGS. Pesquisando cuidadores: visita a uma prática metodológica. In: Karsch UMS, organizador. Envelhecimento com dependência: revelando cuidadores. São Paulo: EDUC; 1998. p. 21-45.

18. Paz AA, Santos BRL. Programas de cuidado de enfermagem domiciliar. Rev Bras Enfermagem. 2003; 56(5):538-41.

19. Caldas CP. Envelhecimento com dependência: responsabilidade e demandas da família. Cad Saúde Pública = Rep Public Health. 2003; 19(3):773-81. 